\title{
Valider la guerre : la construction du régime d'expertise stratégique
}

Validating War: the making of a strategic expertise regime

\section{Christophe Wasinski}

\section{(2) OpenEdition}

\section{Journals}

Édition électronique

URL : http://journals.openedition.org/conflits/17862

DOI : $10.4000 /$ conflits. 17862

ISSN : $1777-5345$

Éditeur :

CCLS - Centre d'études sur les conflits lilberté et sécurité, L'Harmattan

\section{Édition imprimée}

Date de publication : 13 septembre 2010

Pagination : 39-58

ISBN : 978-2-296-12083-9

ISSN : 1157-996X

Référence électronique

Christophe Wasinski, «Valider la guerre : la construction du régime d'expertise stratégique », Cultures \& Conflits [En ligne], 77 | Printemps 2010, mis en ligne le 13 septembre 2011, consulté le 30 mars 2021. URL : http://journals.openedition.org/conflits/17862 ; DOI : https://doi.org/10.4000/conflits. 17862 


\section{Valider la guerre : la construction du régime d'expertise stratégique}

\section{Christophe WASINSKI}

Christophe Wasinski est docteur en sciences politiques (relations internationales), maitre de conférences aux Facultés universitaires de Namur et à l'Université libre de Bruxelles au sein du centre Recherche et Enseignement en politique internationale (REPI). Contact : christophe.wasinski@ulb.ac.be

T a guerre est malheureusement loin d'avoir disparu du paysage international ${ }^{1}$. Après la fin de la guerre froide, les forces armées ont continué à se préparer à ce phénomène comme l'ont bien montré, au cours des années 1990 et au début des années 2000, les projets de Révolution dans les Affaires Militaires ou de Transformation aux Etats-Unis. Plus encore, de nombreuses armées se sont régulièrement impliquées dans des opérations guerrières diverses comme en Irak en 1991, au Kosovo en 1999, en Afghanistan depuis 2001 et encore en Irak depuis 2003. Comme l'ont pourtant noté Keith Krause et Michael C. Williams, de façon étonnante et en dépit de sa prégnance, la violence guerrière reste sous-théorisée par les recherches interprétatives en relations internationales ${ }^{2}$.

L'ambition de cet article est d'apporter une contribution dans ce domaine. Dans cette optique, il s'interroge sur les mécanismes de la pensée stratégique qui ont contribué à faire de la guerre moderne une pratique sociale jugée techniquement faisable et, partant de là, légitime pour les militaires ${ }^{3}$. Pour ce faire, l'analyse proposée s'inscrit dans les champs, très proches sur le plan théorique, de la sociologie pragmatique (en s'inspirant d'auteurs tels que Luc Boltanski, Nicolas Dodier et Francis Chateauraynaud) et de la sociologie des sciences (en s'appuyant surtout sur les travaux de Bruno Latour). D’une part,

1. Nous désirons tout particulièrement remercier Nina Wilen pour ses commentaires pertinents portant sur une version préalable de ce texte ainsi que les deux évaluateurs anonymes de la revue.

2. Krause K., Williams M. C., "From Strategy to Security: Foundations of Critical Security Studies”, in Critical Security Studies - Concepts and Cases, Londres, UCL Press, 1997, p. 51.

3. Tout en admettant que des recherches complémentaires permettraient certainement de bien faire apparaître l'extension de cette légitimité dans d'autres sphères, y compris parmi les civils. 
la sociologie des sciences a développé un très fertile questionnement portant sur la construction des faits scientifiques dont la présente étude s'inspirera largement. D'autre part, la sociologie pragmatique a élaboré un cadre de compréhension des actions collectives qui s'articule à l'enquête sur la construction sociale des faits. Grâce à ces deux approches, nous nous proposons d'enquêter sur la formation d'un régime d'expertise stratégique qui soutient la légitimité technique de l'emploi de la force militaire. Ensemble, la sociologie des sciences et la sociologie pragmatique apportent un éclairage particulièrement pertinent à la question de la guerre.

\section{Décision belliqueuse et registres justificatifs}

Les tentatives d'explication du phénomène guerrier ne manquent pas, loin s'en faut ${ }^{4}$. Néanmoins, ce que passent assez largement sous silence beaucoup d'explications traditionnelles (comme celles qui insistent sur la recherche de territoire, de matière première ou encore sur la volonté de puissance), c'est le détail du processus intersubjectif par lequel les acteurs produisent des représentations communes relatives à leur situation, leurs problèmes ou encore sur les solutions envisageables pour affronter ceux-ci, et qui les mènent finalement sur le sentier de la guerre 5 . Effectivement, la guerre, en tant qu'action sociale concertée et organisée n'est possible que s'il existe à l'origine un consensus, aussi minime, fragile ou fragmentaire soit-il, sur la décision de sa mise en œuvre. Pour saisir l'action politique qu'est la guerre, il conviendrait alors de s'interroger sur les grands registres d'argumentation (juridique, moral, diplomatique, métaphorique, etc.) qui la justifient et permettent d'arriver à ce fameux consensus ${ }^{6}$. De fait, si l'on prend le temps d'analyser avec un minimum d'attention un conflit international parmi d'autres, on repèrera habituellement que des explications multiples aux raisons de partir en guerre, tour à tour apportées par les diplomates, les officiers, les hommes politiques et autres experts, sont en présence. Partant de là, on peut assez raisonnablement affirmer que la décision belliqueuse finale reposera sur l'agrégation, l'articulation et/ou la hiérarchisation de plusieurs de ces registres. En guise d'exemple, dans le cas de la guerre de 2003 contre l'Irak, on remarquera que l'existence de soidisant « armes de destruction massive » servit de point focal autour duquel

4. Suganami H., "Bringing Order to the Cause of Wars Debates", Millennium: Journal of International Studies, vol. 19, n¹, 1990, pp. 19-36.

5 . Les approches cognitives, sociales constructivistes et poststructuralistes ont cependant défriché le terrain en ce qui concerne la production de représentations menant à des politiques belliqueuses. Voir par exemple : Lindemann T., Penser la guerre - L'apport des constructivistes, Paris, L'Harmattan, 2008 ; Campbell D., Writing Security - United States Foreign Policy and the Politics of Identity, Minneapolis, University of Minnesota Press, 1998 ; Shapiro M. J., Violent Cartographies - Mapping the Cultures of War, Minneapolis et Londres, University of Minnesota Press, 1997 ; Jervis R., "War and Misperception", Journal of Interdisciplinary History, vol. $18, \mathrm{n}^{\circ} 4$, printemps 1988 , pp. 675-700.

6. Dans ce qui suit, nous nous inspirons de : Boltanski L., De la justification - Les économies de grandeur, Paris, Gallimard, 1991 ; Latour B., La science en action - Introduction à la sociologie des sciences, (trad. de l'anglais), Paris, La Découverte, 2005. 
s'organisèrent des justifications mettant en cause la personne de Saddam Hussein, l'obligation morale d'agir ou encore des liens suspectés entre le régime irakien et $\mathrm{Al}$ Qaeda ${ }^{7}$. En résumé, les décideurs négocient entre eux un consensus qui s'exprime par des justifications ${ }^{8}$.

Dans le présent article, il a été choisi d'analyser la construction d'un seul de ces registres justificatifs ; celui qui ne cesse d'affirmer en toutes circonstances que «la guerre, c'est techniquement faisable! » Ce registre est basé sur l'existence d'une vaste communauté interprétative qui a non seulement contribué à la constitution des forces armées nationales, donnant aux guerres étatiques leur forme distinctive, mais qui a aussi, et surtout, rendu les conflits armés techniquement pensables 9. C'est ce point d'appui conventionnel, incontournable à toute décision belliqueuse, mais aussi à d'autres choix, tels que ceux qui concernent la fabrication des armements ou la confection des budgets de défense, que nous avons considéré comme un " régime d'expertise stratégique »10. Les recherches journalistiques sur la guerre d'Irak de 2003 exposent encore particulièrement bien la façon dont ce régime d'expertise est incorporé dans des négociations préalablement au conflit ${ }^{11}$. Elles illustrent la façon dont les militaires et les dirigeants civils, en discutant des plans de guerre et de leur faisabilité, ont mobilisé et adapté ce régime d'expertise duquel résultera l'opération Iraqi Freedom.

Qu'il soit cependant bien clair que ce n'est pas le problème de l'efficacité «physique » ou «matérielle » des pratiques stratégiques qui nous préoccupe ${ }^{12}$. C'est bel et bien la question de savoir par quelles dynamiques la foi en la faisabilité technique de la guerre s'est imposée. Autrement dit, pour paraphraser une expression courante dans le langage des politologues, on cherchera ici à mieux comprendre certains des mécanismes par lesquels l’idée stratégique, qui sous-tend historiquement l'institution militaire, est parvenue à s'imposer et se maintenir « à l'agenda » social. Par ce biais, le texte qui suit est

7. The White House, "President Bush Outlines Iraqi Threat", 7 octobre 2002 (www.gwu.edu/ nsarchiv/NSAEBB/NSAEBB80/new/doc\%2012/President $\% 20 B u s h \% 20$ Outlines\%20Iraqi\%20Threat.htm).

8. Le travail classique de Allison G. T., Essence of Decision - Explaining the Cuban Missile Crisis, Harvard, Harper, 1971, donne déjà une illustration de ce processus de négociations.

9. Dodier N., "Les appuis conventionnels de l'action - Eléments de pragmatique sociologique", Réseaux, novembre-décembre 1993, n62, pp. 63-86. Dans le domaine des études de sécurité, voir : Wasinski C., "Produire de la capacité de gestion de crise internationale : le cas de l'OTAN pendant les années 2000", Cultures E Conflits, n75, hiver 2009, pp. 15-31; Dumoulin A., Wasinski C., "Justifier l'arme nucléaire. Le cas français pendant les années 1990”, Etudes internationales, vol. XLI, n¹, mars 2010, pp. 79-96..

10. Dodier N., L'expertise médicale - Essai de sociologie sur l'exercice du jugement, Paris, Métailié, 1993.

11. Hersh S., Dommages collatéraux, (trad. de l'américain), Paris, Gallimard, 2006 ; Woodward B., Plan d'attaque (trad. de l'américain), Paris, Denoël, 2004.

12. Sur cette distinction, voir aussi : Latour B., Sur le culte moderne des dieux faitiches (suivi de Iconoclash), Paris, Les empêcheurs de penser en rond / La Découverte, 2009 ; Hacking I., Entre science et réalité - La construction sociale de quoi ? (trad. de l'anglais), Paris, La Découverte, 2001. 
donc également une contribution à l'interrogation qui porte sur la normalisation de l'emploi de la force militaire grâce à la diffusion d'une idéologie de la faisabilité 13 .

Sur le fond, le cœur du régime d'expertise stratégique se trouve dans la pensée stratégique des militaires, lieu d'affirmation par excellence de cette faisabilité technique ${ }^{14}$. Plus spécifiquement encore, dans cette analyse, nous avons décidé de centrer l'étude sur l'analyse des discours contenus dans la pensée stratégique écrite. Il est néanmoins évident qu'il existe une réflexion stratégique orale. Mais l'aspect structurant de l'écrit, dans des sociétés européanisées qui accordent tant d'importance aux textes sous forme de constitutions, lois, règlements, ou encore procédures, légitime le fait de se concentrer sur l'écrit (dès lors aussi que l'on accepte qu'il n'épuise pas complètement le sujet de la construction du régime d'expertise stratégique). Au final, par l'analyse des écrits, il est possible de mettre en évidence non pas une « simple » narration historique mais, ce qui nous intéresse primordialement, des mécanismes plus ou moins généraux qui contribuent largement à bâtir et à assurer la reproduction de ce fameux régime d'expertise stratégique ${ }^{15}$. Ajoutons encore que, pour des raisons d'espace, nous avons dû restreindre l'étendue des discours pris en considération ${ }^{16}$. Nous nous sommes concentrés sur la pensée militaire terrestre produite en Europe et aux Etats-Unis de la fin du Moyen Age à nos jours, une fois encore, pour son pouvoir structurant. Nous avons également décidé de ne pas prendre en considération le champ de la guérilla, de la guerre révolutionnaire, de la lutte contre-insurrectionnelle, etc. qui est habituellement construit par les acteurs comme une expertise différenciée de l'expertise stratégique dite « classique ». Par ailleurs, nous ne ferons qu'effleurer le champ stratégique nucléaire pour des raisons similaires.

13. Questionnement central de l'approche dite de culture stratégique. Johnston A.I., Cultural Realism - Strategic Culture and Grand Strategy in Chinese History, Princeton, Princeton University Press, 1995. Sur la normalisation et l'anormalisation, voir bien entendu : Foucault M., Naissance de la clinique, Paris, PUF, 2003 ; et Histoire de la folie à l'âge classique, Paris, Gallimard, 1972.

14. Comme on le verra, nous nous distancions cependant des travaux « classiques » des études de sécurité portant sur la pensée stratégique et les doctrines militaires (Kier, Posen, Snyder, etc.) entre autres, car ces dernières considèrent le plus souvent l'existence de l'institution militaire comme une variable indépendante. Pour une synthèse de ces approches, voir : Wasinski C., “Théories des relations internationales, doctrines militaires et pensée stratégique et de sécurité", Les Champs de Mars, n¹4, deuxième semestre 2003, pp. 145-172.

15. En ce sens, cette analyse n'a aucune prétention en matière de description historique de la pensée stratégique. En guise d'introduction à l'histoire de cette pensée, voir par exemple : Gat A., A History of Military Thought from Enlightenment to the Cold War, Oxford, Oxford University Press, 2001; Coutau-Bégarie H., Traité de stratégie, Paris, Economica - ISC, 1999.

16. Notons également qu'outre les ouvrages historiques cités en note de bas de page tout au long de l'article, notre recherche nous a amené à consulter un corpus de plus d'une centaine de textes militaires anciens (du XVI e siècle à 1945). Pour des raisons d'espace, ces ouvrages ne peuvent être cités. Ils ont cependant eu un rôle essentiel dans le développement du raisonnement. 


\section{Les composantes du cadre interprétatif stratégique classique}

Le régime d'expertise stratégique apparaît entre la fin du Moyen Age et le XVII siècle. C'est à ce moment que se constitue, de façon progressive, un cadre interprétatif relatif à la façon de mener des guerres modernes à partir de l'expérience tirée des très nombreux conflits qui opposent les entités politiques à travers toute l'Europe (telles que les guerres de Louis XIV). On soulignera d'emblée que le savoir qui constitue ce cadre contribue largement à donner forme à la guerre moderne, très différente de celle que l'on rencontre au Moyen Age, en fondant une représentation bien plus organisée et décisive de l'utilisation de la violence. Ce faisant, ce savoir contribue à définir ce que l'on entend lorsque l'on emploie le mot « guerre » dans l'univers étatique moderne. Il importe donc de s'interroger sur les modes d'inscription, d'objectivation et de transmission de ce même savoir. Par ce biais, on comprendra mieux de quelle façon les acteurs confèrent, de proche en proche, par rassemblement progressif d'éléments épars, du " micro au macro », une existence publique et un sens socialement accepté à la technique guerrière 17 .

Pendant la période considérée, la formation du régime d'expertise stratégique prend sa source dans des dizaines de traités militaires techniques et tactiques, aujourd'hui bien recensés par des historiens militaires, qui mettent en évidence le retour de l'infanterie déployée dans des dispositifs organisés ${ }^{18}$. Le plus célèbre de ces textes est sans conteste L'Art de la guerre de Nicolas Machiavel. Dans ce domaine, le texte du Florentin côtoie cependant de nombreux traités rédigés par des officiers européens bien moins connus de nos jours (Billon, de Gheyn, Rohan, Wallhausen). On retrouve dans ces textes, pour reprendre les concepts de Michel Foucault, une accumulation de savoirs de type disciplinaires centrés sur le soldat individuel ou en groupe(s) (voir également la représentation $\mathrm{n}^{\circ} 1$ disséquant le comportement d'un soldat manipulant une arme à feu) ${ }^{19}$. Ils contiennent effectivement nombre d'informations sur la façon dont les hommes doivent être entraînés, déployés ou déplacés dans des formations bien organisées.

17. Chateauraynaud F., “Forces et faiblesses de la nouvelle anthropologie des sciences”, Critique, juin-juillet $1991, n^{\circ} 529-530$, p. 463.

18. Kleinschmidt H., "Using the Gun: Manual Drill and the Proliferation of Portable Firearms", The Journal of Military History, vol. 63, n³, juillet 1999, pp. 601-629 ; Gonzales de Leon F., "'Doctors of the Military Discipline': Technical Expertise and the Paradigm of the Spanish Soldier in the Early Modern Period”, Sixteenth Century Journal, vol. 27, n¹, printemps 1996, pp. 61-85 ; Hale J. R., War and Society in Renaissance Europe, 1450-1620, Baltimore, Hopkins University Press, 1985.

19. Foucault M., Surveiller et punir - Naissance de la prison, Paris, Gallimard, 1975. 


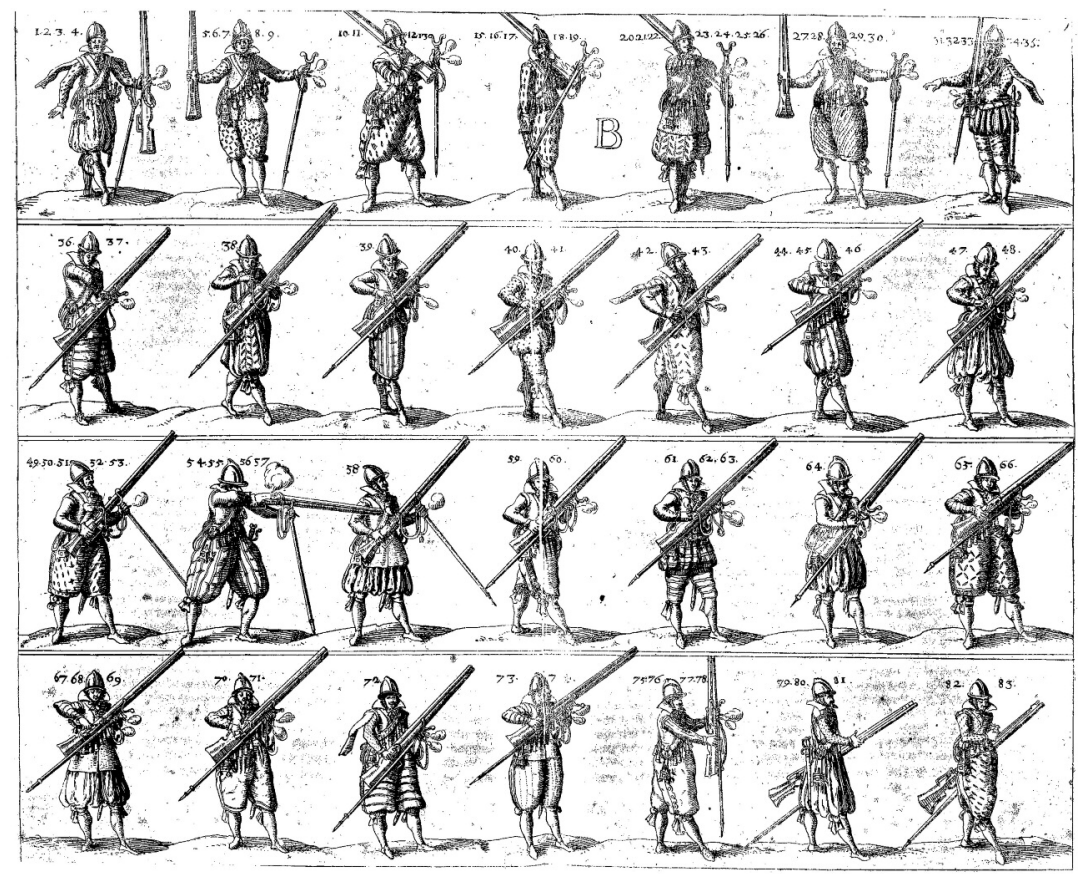

1. Johann Wallhausen, L'art militaire pour l'infanterie, (trad. de l'allemand), Franeker, Balck, ca 1615, s.p. (représentation intercalée entre les pages 8 et 9 - consulté sur gallica.bnf.fr).

On y retrouve aussi un vocabulaire relatif aux fonctions militaires et à la hiérarchie (on y parle de piquiers, de mousquetaires, de chefs de file, d'officiers, etc.). Comme les historiens militaires l'on montré, l'ensemble a d'indéniables effets interpellant (ou performatifs) dans la construction des soldats dont le rôle clairement défini dans ces textes devient un idéal normalisateur 20. Ce savoir produit finalement des représentations réifiées des soldats déployés sur les champs de bataille en ordres carrés ou rectangulaires (voire, parfois, dans des formes plus fantaisistes qui n'ont cependant pas toujours été effectivement testées en campagne) ${ }^{21}$. Ces dispositifs confèrent une grande cohésion aux militaires car ils y sont soumis à un étroit contrôle de leurs pairs, ce qui les contraint à aller de l'avant. Les hommes ainsi surveillés sont alors capables de mener de dangereuses batailles d'anéantissement en lieu et place d'opérations de harcèlement qui semblent avoir été l'apanage des combattants du Moyen Age. Selon plusieurs recherches contemporaines, bien que cette capacité d'anéantissement ne s'actualise pas de la même manière à toutes les époques, il s'agit là d'une étape fondamentale dans le développement d'une culture guerrière centrée sur la destruction physique de l'ennemi ${ }^{22}$.

20. De façon plus générale, sur ce type de dynamique, voir : Butler J., Gender Trouble Feminism and the Subversion of Identity, London, Routledge, 1999.

21. Jones R. W., Security, Strategy, and Critical Theory, Colorado, Lynne Rienner, 1999. 
La formation du cadre interprétatif repose ensuite sur une mise en forme particulière du savoir technique militaire. La crédibilité de ce savoir ne s'appuie effectivement pas uniquement sur son contenu, mais aussi sur la façon dont ce dernier est exprimé dans les traités militaires qui voient le jour à partir de la fin du Moyen Age. (a) Tout d'abord, il s'agit d'un savoir qui est formalisé sous forme imprimée ${ }^{23}$. Le recours à ce médium, largement favorisé par l'apparition de la presse au XVI e siècle, est un moyen de stabiliser les contenus (la parole ayant la réputation d'être plus souple et plus déformable) et de rendre la connaissance détachable de l'expérience corporelle et du contexte social immédiat ${ }^{24}$. La codification écrite du savoir militaire participe aussi à une logique de renforcement bureaucratique (qui contribue elle-même au développement des Etats européens au sortir du Moyen Age) 25. On gardera cependant à l'esprit qu'en dépit de sa puissance et de son pouvoir structurant, il ne faut pas ipso facto considérer que la pensée écrite épuise le domaine stratégique. (b) L’écriture utilisée dans les traités assume tous les atours de la technicité. Ce n'est pas une écriture métaphorique, poétique ou romanesque; le plus souvent, c'est une écriture précise, structurée et, sommes-nous tentés d'ajouter, laborieuse à la lecture. On remarquera aussi que la plupart des titres des traités sont de nature rhématique ${ }^{26}$. Effectivement, les titres renvoient directement au statut des ouvrages qui sont Théorie de la guerre, Art de la guerre, Mémoire sur la guerre, etc. La transparence de ces titres prouve leur volonté d'appartenir à un genre, à une communauté de sens. (c) Le format des traités est par ailleurs très inspiré par le savoir des ingénieurs qui produisent plans de forteresses et autres cartes de campagnes pour les militaires ${ }^{27}$. En fait, les ingénieurs lèguent aux militaires une pensée " géométrisante » qui sert de fil conducteur narratif spatial ; les actions militaires sont racontées comme des événements qui se succèdent (non pas dans les consciences mais) dans l'espace, éventuellement sur un plan ou sur une carte (voir ainsi la reproduction $n^{\circ} 2$ exposant le déploiement d'unités militaires en «ordre oblique » sur un champ de bataille). Ce géométrisme ambiant est aussi repérable par le recours à un vocabulaire très spatialisé (front, flanc, avant-garde, etc.). L'ensemble permet de mettre en ordre le chaos de la bataille et de rendre racontable et compré-

22. Hanson V. D., Le modèle occidental de la guerre - La bataille d'infanterie dans la Grèce classique, (trad. de l'américain), Paris, Les Belles Lettres, 1990 ; Joxe A., Voyage aux sources de la guerre, Paris, PUF, 1991 ; Keegan J., A History of Warfare, Londres, Pimlico, 1993.

23. MacLuhan M., La Galaxie Gutenberg face à l'ère électronique - les civilisations de l'âge oral à l'imprimerie, (trad. de l'anglais), Paris, Marne, 1967.

24. Certeau M. de, L'écriture de l'histoire, Gallimard, Paris, 1975, pp. 243-335 et pp. 229-231; Barthes R., Le degré zéro de l'écriture, Paris, Seuil, 1972, p. 18.

25. Tilly C. (dir.), The Formation of National State in Europe, Princeton, Princeton University Press, 1975.

26. Genette G., Seuils, Paris, Seuil, 1987.

27. Pollak M. D., Military Architecture Cartography \& the Representation of the Early Modern European City - A Checklist of Treatises on Fortification in the Newberry Library, Chicago, The Newberry Library, 1991; Duffy C., The Fortress in the Age of Vauban and Frederick the Great 1660-1789 - Siege Warfare volume II, Londres, Routledge \& Kegan Paul, 1985 ; et Siege Warfare - The Fortress in the Early Modern World 1494-1660, Londres, Routledge \& Kegan Paul, 1979. 
hensible (ou de «mettre en intrigue », pour reprendre l'expression de Paul Ricœur) une multitude d'événements se déroulant en parallèle ${ }^{28}$. On constatera cependant que le cadre géométrisant naturalise l'existence d'un point de vue narratif au détriment d'autres formes de narrations. Ainsi, les narrations de la guerre qui assument leur part de subjectivité (comme de nombreux récits de nature autobiographique dans lesquels le vécu des hommes sur le terrain est raconté) ne sont guère prises en considération. Le point de vue "cartographique » de celui qui surplombe de haut le champ de bataille semble s'imposer comme seul regard important réellement légitime ${ }^{29}$. Cette remarque s'avère par ailleurs pertinente dans le domaine de l'histoire militaire « classique » (dite « histoire bataille»). En fait, il faut considérer l'histoire militaire écrite par les officiers et pour les officiers comme une partie intégrante de la pensée stratégique (en ce sens, le genre se démarque nettement de l'écriture des anciens mémoires militaires trop peu précis sur le déroulement des actions militaires) ${ }^{30}$. La structure narrative de ces écrits et les dynamiques encadrant leur développement évoluent en fait de manière très similaire à celles de la pensée stratégique théorique classique.

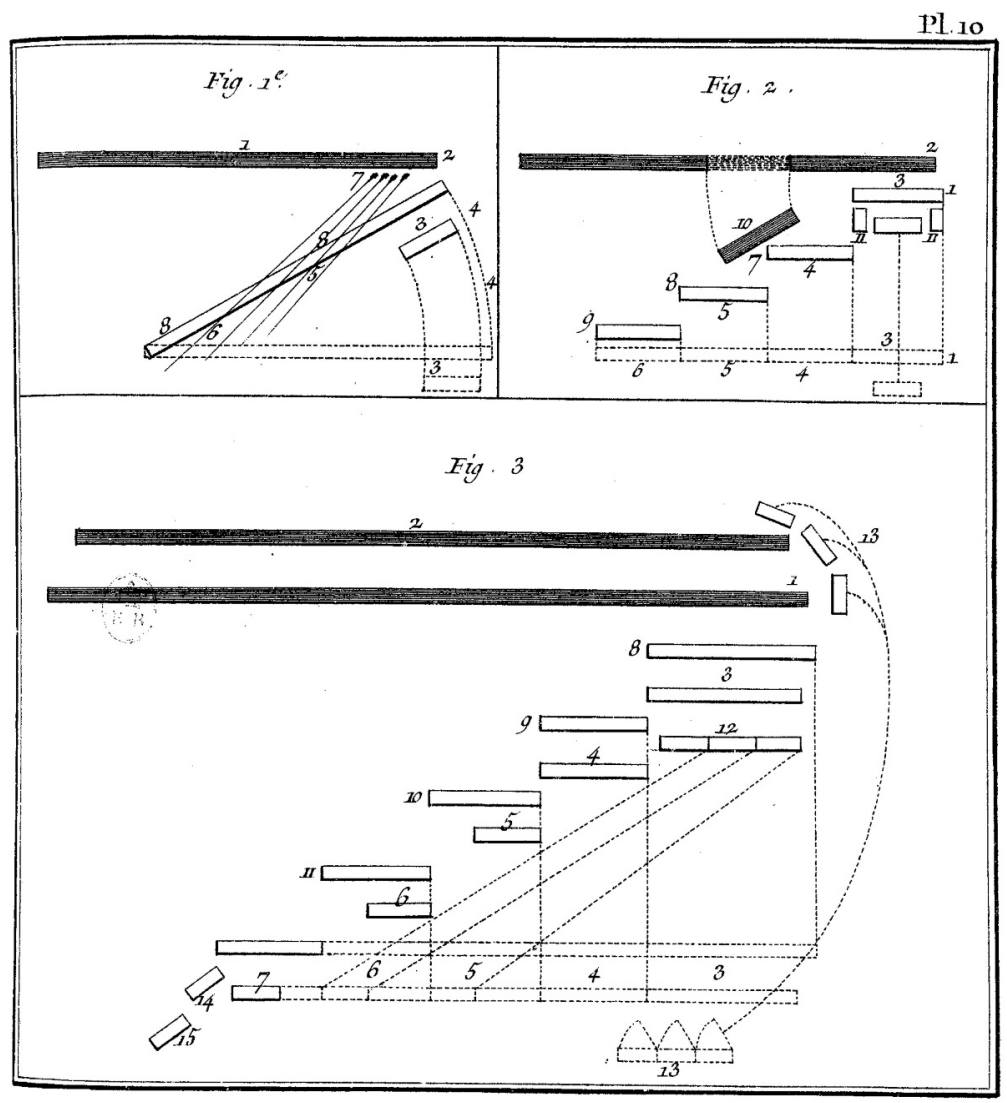

2. Chevalier de Grimoard, Essai théorique et pratique sur les batailles, Paris, Dessaint, 1775, sp (consulté sur gallica.bnf.fr). 
Enfin, la dynamique textuelle évoquée joue un rôle fondamental dans l'établissement d'un grand partage entre politique et technique ${ }^{31}$. La pensée militaire participe à la construction d'une dichotomie entre, d'une part, une sphère politique responsable de la décision finale relative à l'emploi de la violence et, d'autre part, une sphère technique régie par des principes militaires auquel il est « impossible» de déroger (voir également infra). On peut bien entendu voir dans ce processus un élément qui marque la différenciation de sphères d'activités sociales dans une logique durkheimienne ${ }^{32}$. Mais, au-delà de cet aspect, il faut aussi constater que la pensée militaire pratique un coup de force ; elle s'arroge (au moins partiellement) le droit de déterminer la limite d'intervention du politique dans l'acte guerrier considérant que la spécificité de son action s'impose de façon universelle (de manière très simplifiée : "le politique a le droit de déclencher le conflit mais n'a rien à dire quant à son déroulement sur le champ de bataille qui dépend de règles que seuls les militaires maîtrisent "). Il y a indubitablement un aspect paradoxal dans tout ceci; le militaire, en même temps qu'il répète sa soumission «sincère et totale » au politique, cherche à imposer une délimitation à cette soumission. On l'aura compris, ceci nous fait entrer de plain pied dans le discours de Clausewitz et, probablement plus encore, dans les discours sur Clausewitz (par exemple, les interprétations allemandes édifiantes de Moltke et Ludendorff) ${ }^{33}$. Ceux-ci renvoient encore et encore à la question du tracé de la frontière entre politique et militaire... telle qu'elle est majoritairement établie par les militaires. Ces débats servent aussi, bien entendu, à conférer une légitimité utilitaire à la guerre en faisant d'elle un outil incontournable du politique.

En définitive, le cadre interprétatif fournit des prises assez largement acceptées à partir desquelles les stratégistes des siècles à venir pourront, comme on va le voir, mettre en branle un travail intertextuel de maillage et une institutionnalisation plus poussée ${ }^{34}$.

28. Ricœur P., Temps et récit, 3 tomes, Paris, Seuil, 1983-1985. Sur la construction des récits, voir aussi : Lyotard J-F., La condition postmoderne, Paris, Minuit, 1979.

29. Panofsky E., La perspective comme forme symbolique, (trad. de l'allemand), Paris, Minuit, 1975.

30. Bloom J., "History, Military", in Margiotta F. D. (dir.), Brassey's Encyclopedia of Military History and Bibliography, (préf. de John Keegan), Washington et Londres, Brassey's, 1994, pp. 475-497 ; Cox G. P., "Of Aphorisms, Lessons, and Paradigms: Comparing the British and German Official Histories of the Russo-Japanese War”, The Journal of Military History, vol. 56, n³, juillet 1992, pp. 389-402; Harari Y. N., Renaissance Military Memoirs - War, History and Identity, 1450-1600, Boydell Press, Woodbridge, 2004.

31. Nous empruntons cette notion à Latour B., Nous n'avons jamais été modernes - Essai d'anthropologie symétrique, Paris, La Découverte, 1991, dans sa relecture de : Schaffer S., Shapin S., Léviathan et la pompe à air - Hobbes et Boyle entre science et politique, Paris, La Découverte, 1993.

32. Durkheim E., De la division du travail social, Paris, PUF, 1986.

33. Aron R., Penser la guerre - Clausewitz, 2 vol., Paris, Gallimard, 1976. Sur Ludendorff, voir aussi infra. Les commentaires sur Clausewitz abondent dans la littérature portant sur la pensée stratégique et son histoire. Pour cette raison, nous préférons ici nous abstenir d'aller plus loin sur cet auteur pour davantage nous consacrer à des éléments habituellement laissés en jachère dans le champ des études stratégiques et de sécurité. 


\section{Confirmer l'efficacité militaire}

Les bases du cadre stratégique classique posées ne doivent pas laisser penser que la pérennité du régime d'expertise serait acquise une fois pour toutes ; ce régime doit incessamment être réactivé pour continuer à influer sur le cours des événements. En d'autres termes, les stratégistes doivent se battre pour maintenir à flot dans les esprits la conviction selon laquelle «la stratégie, ça marche ». Pour ce faire, un travail discursif de répétition et de confirmation est rapidement entrepris par la communauté des stratégistes. Comme le montre l'histoire de la pensée stratégique, c'est un travail qui a (entre autres) été particulièrement bien mené du XVII ${ }^{\mathrm{e}}$ au XIX ${ }^{\mathrm{e}}$ siècle à partir d'ouvrages concernant surtout les guerres de Frédéric II de Prusse, les guerres napoléoniennes ou encore les guerres d'unification allemande ${ }^{35}$. Quatre modalités doivent en particulier être mises en évidence à ce sujet :

De façon générale, le régime d'expertise stratégique se construit largement à partir du cadre selon un mécanisme d'intertextualité ${ }^{36}$. Selon cette dynamique, le renforcement s'opère de manière capillaire, couche textuelle après couche textuelle, par l'emprunt et la reprise d'idées. En fait, la plupart des auteurs trouvent à citer leurs pairs en de nombreuses occasions. C'est une pratique qui s'impose avec la fin du Moyen Age. Durant cette période, les textes s'organisent de façon hiérarchique par rapport à un « point central » qui est la Bible. C'est ce point central qui fonde l'autorité, et donc aussi la crédibilité, la véracité et la réalité, de tous les autres écrits ${ }^{37}$. Après cette période, l'autorité biblique s'efface dans de nombreux domaines. L'autorité ultime ne repose

34. Sur la notion de prise, voir : Bessy C., Chateauraynaud F., Experts et faussaires - Pour une sociologie de la perception, Paris, Métaillié, 1995.

35. Pour plus d'informations historiques, voir : Queloz D., De la manoenvre napoléonienne à l'offensive à outrance - La tactique générale de l'armée française 1871-1914, Paris, Economica - ISC, 2009 ; Colson B., Le général Rogniat, ingénieur et critique de Napoléon, Paris, Economica - ISC, 2006 ; Eysturlid L. W., The Formative Inluences - Theories, and Campaigns of the Archiduke Carl of Austria, Londres, Greenwood, 2000 ; Colson B., Coutau-Bégarie H. (dir.), Pensée stratégique et humanisme - De la tactique des Anciens à l'éthique de la stratégie, Paris, Economica / ISC / FUNDP, 2000 ; Colson B., L'Art de la guerre de Machiavel à Clausewitz, Namur, Presses Universitaires de Namur, 1999 ; Colson B., La culture stratégique américaine - L'influence de Jomini, Paris, FEDN - Economica, 1993 ; Duffy C., The Military Experience in the Age of Reason, New York, Atheneum, 1988 ; Chagniot J., Le chevalier de Folard - La stratégie de l'incertitude, Monaco, Editions du Rocher, 1997 ; Paret P. (dir.), Makers of Modern Strategy from Machiavelli to the Nuclear Age, Oxford, Clarendon Press, 1986 ; Poirier L., Les voix de la stratégie, généalogie de la stratégie militaire - Guibert, Jomini, Paris, Fayard, 1985 ; Rothenberg G. E., The Art of Warfare in the Age of Napoleon, Bloomington et Londres, Indiana University Press, 1981 ; Earle E. M. (dir.), Les maîtres de la stratégie, 2 vol., (trad. de l'américain), Paris, Flammarion, 1980 ; Ross S. T., From Flintlock to Rifle - Infantry Tactics, 1740-1866, Londres, Cass, 1979 ; Paret P., Clausewitz and the State, New York, Oxford University Press, 1976 ; Howard M. (dir.), The Theory and Practice of War, London, Cassel, 1965 ; Carrias E., La pensée militaire française, Paris, PUF, 1960 ; Carrias E., La pensée militaire allemande, Paris, PUF, 1948 ; Caemmerer R. von, L'évolution de la stratégie au XIXe siècle, (trad. de l'allemand), Paris, Fischbacher, 1907.

36. Kristeva J., Semeiotike - Recherche pour une sémanalyse, Paris, Seuil, 1969.

37. Compagnon A., La seconde main ou le travail de la citation, Paris, Seuil, 1979. 
plus sur un "point central », mais plutôt sur un réseau complexe de références. Cet assemblage peut d'ailleurs être décortiqué pas à pas en rendant compte de quel officier s'appuie sur quel autre officier dans les ouvrages fondant le régime d'expertise stratégique. En lisant Feuquières, on retrouve les noms de Montecuculli, Folard, Coehoorn, Vauban, etc. En lisant Maizeroy, on rencontre les noms de Montecuculli, Feuquières, Puységur, Mesnil-Durand, de Saxe, etc. En lisant Grimoard, on rencontre les noms de Montecuculli, Puységur, Guischardt, Maizeroy, etc. En lisant Mesnil-Durand, on croise Montecuculli, Folard, Feuquières, Puységur, Guischardt, Maizeroy, etc. Puységur, Feuquières, Folard, Montecuculli sont cités par Bosroger. L'énumération de ces grands noms, dont la plupart sont pourtant aujourd'hui oubliés, qui ont pensé les dispositifs adéquats face à la généralisation des armes à feu et réfléchi aux déploiements militaires européens du XVII au XVIII siècle, peut s'arrêter ici ; on l'aura compris, le système de référencement est dense. Ce système contribue à donner de la crédibilité aux assertions militaires techniques contenues dans les ouvrages et est à l'origine d'une véritable communauté interprétative ${ }^{38}$. On notera aussi que les connexions intertextuelles reposent sur une double dynamique. (a) D'une part, les connexions sont le fait d'une démarche individuelle. Les stratégistes (souvent, mais pas obligatoirement, des militaires) ont tendance à se montrer «calculateurs » en faisant en sorte que leurs textes coïncident avec le style défini par le cadre et qu'ils correspondent le plus possible aux attentes supposées du régime d'expertise. Ce point a par exemple été bien exposé dans les travaux biographiques contemporains portant sur l'historien et théoricien militaire Antoine de Jomini (1779-1869), longtemps considéré comme l'un des plus grands interprètes des opérations napoléoniennes ${ }^{39}$. Ils maximisent ainsi la chance d'être pris en considération par leurs pairs et, de cette manière, sont plus facilement intégrés dans l'édifice stratégique. (b) D'autre part, l'intégration au sein de l'édifice passe aussi par l'acceptation, par les pairs, du texte produit par tel ou tel stratégiste ${ }^{40}$. Il s'agit là d'une épreuve que doit subir chaque texte pour faire partie du régime d'expertise et se voir assigner une place dans la structure intertextuelle. En fait, cette seconde étape, qui renvoie à la liste d'auteurs ci-dessus, est certainement plus importante que la première car, bien que l'on puisse tenter de s'imposer comme un expert par une démarche volontariste, le dernier mot sur le statut appartient néanmoins à l'édifice intertextuel et, plus globalement, à la place que l'œuvre se voit historiquement attribuée.

38. Fish S., Quand lire c'est faire - L'autorité des communautés interprétatives, (trad. de l'anglais), Paris, Les Prairies ordinaires, 2007.

39. Langendorf J-J., Faire la guerre: Antoine-Henri Jomini, 2 vol., Genève, Georg, 2004. On trouvera un exemple plus recent dans : Mearsheimer J. J., Liddell Hart and the Weight of History, New York, Brassey's, 1988.

40. A ce propos, voir aussi : Foucault M., "Qu'est-ce qu'un auteur ?” in Dits et écrits, 1954-1975, vol. I, Paris, Gallimard, 2001, pp. 817-837. 
La dynamique intertextuelle se déploie également à travers un double processus de désingularisation et de résingularisation partielle (que l'on peut aussi appeler «traduction » ${ }^{41}$. (a) Le régime d'expertise stratégique a l'ambition de créer un savoir technique formel qui a une prétention de validité qui dépasse le cas individuel. Pour parvenir à cet effet, il est contraint de procéder en désingularisant chaque événement. C'est par ce biais qu'un penseur aussi important que Jomini est en mesure de comparer une guerre du XVIII siècle à une guerre du XIXe siècle (et inversement), alors que ce ne sont plus les mêmes hommes, plus les mêmes motifs, plus les mêmes lieux qui sont évoqués, et ensuite d'établir des «principes de la guerre » dont la validité est considérée comme universelle ${ }^{42}$. La désingularisation permet non seulement de produire de la comparabilité, mais sert aussi de point d'appui à une pensée stratégique qui se veut scientifique et donc fondée sur des récurrences prévisibles. (b) Dans un second temps, cependant, on assiste à un travail de résingularisation. Chaque stratégiste s'inspire certes de ce que ses pairs ont dit, mais il ne répète pas mot pour mot la même chose que ceux-ci. Il dispose d'une certaine latitude qui lui permet de réintroduire du singulier dans la désingularisation. C'est en particulier par ce processus que l'édifice intertextuel a été à même de rendre compte et d'encourager le changement d'échelle de la guerre. La dilatation des espaces de combat et l'augmentation des effectifs militaires vont être progressivement traduites dans la pensée militaire sans contestation réelle du cadre stratégique de base. Ce cadre est adapté grâce à l'utilisation du même vocabulaire, des mêmes concepts, des mêmes conceptions. D'autres termes sont bien entendu élaborés, et ensuite intégrés à l'édifice, en parallèle : on parle par exemple de grande tactique, d'art opératif, d'opératique ou encore de niveau opérationnel de la guerre pour désigner ce qui s'intercale entre la tactique et la stratégie. Mais il s'agit plus d'adaptation que de remise en question et la pensée vise toujours le moyen d'imposer sa volonté par un emploi décisif de la force contre l'ennemi. On notera par ailleurs que, dans ce processus, le détachement par rapport au corps du soldat est de plus en plus important; les individus anonymes des premiers traités disciplinaires ont cédé la place à des groupes génériques qui sont également réifiés (on représente entre autres ces groupes sur des schémas cartographiques de plus en plus globaux). Il est souvent difficile de ne pas percevoir un effet d'autonomisation de langage dans ce processus qui ne semble plus guère tenir compte du vécu des hommes sur le terrain et renvoie plutôt au vécu d'officiers évoluant dans un quartier général loin du front ${ }^{43}$. Finalement, cette dynamique (que l'on peut suivre pas à pas dans les écrits de Bülow, Lloyd, Jomini, Clausewitz, Moltke, Schlieffen, Colin, Camon, etc.) participe à la marche vers la guerre totale.

41. Dodier N., L'expertise médicale - op.cit., pp. 48, 91 et suiv., 267, 269 ; Callon M., "Eléments pour une sociologie de la traduction - La domestication des coquilles Saint-Jacques et des marins pêcheurs dans la baie de Saint-Brieuc", L’Année sociologique, 1986, vol. 36, pp.169208.

42. Jomini n'est pas l'inventeur de ces principes mais il renforce beaucoup la légitimité accordée à ceux-ci. Alger J. I., The Quest for Victory - The History of the Principles of War, Westport et Londres, Greenwood, 1982. 
La dynamique intertextuelle prend ensuite appui sur l'institutionnalisation des forces armées. Depuis la fin du Moyen Age, les forces armées se sont transformées en de solides institutions bureaucratiques et hiérarchisées. C'est toutefois au cours du XIXe siècle que ce processus atteint sa pleine maturité ${ }^{44}$. La nature intertextuelle du processus ne disparaît pas pour autant dans ce contexte, loin de là. En fait, si les discours stratégiques confèrent une justification à ces institutions, l'inverse est également vrai ; l'importance des discours se trouve renforcée par la présence des institutions. En d'autres termes, l'institution devient un relai pour soutenir le maillage du savoir expert ${ }^{45}$. Partant, les armées modernes ne cessent de répéter leur foi en la valeur technique de la violence à travers des règlements ou des manuels techniques, tactiques et opérationnels, de textes spécialement dédiés aux formations dispensées dans les nouvelles écoles pour officiers (l'un des plus célèbres est The Operations of War Explained and Illustrated de Bruce Hamley qui sera réédité à de nombreuses occasions et servira d'ouvrage de référence non seulement en GrandeBretagne, mais également aux Etats-Unis) ou encore dans les nombreuses revues militaires qui commencent à apparâttre au XIXe siècle (la MilitärWochenblat ; la Militär-Literatur-Zeitung (1819) ; le Zeitschrift für Kunst, Wissenschaft und Geschichte des Krieges (1822); le Bulletin des Sciences Militaires (1824) ; le Journal des sciences militaires (1825) ; le Spectateur militaire (1826-29, 1842-43) ; la Revue militaire - Journal des Armées de Terre et de Mer (1833-1834); la France militaire (1845); la Preussische Webr-Zeitung (1848) ; le Moniteur de l'armée (1840-41, 1847, 1849-1850) ; la Revue bibliographique militaire (1852); le Bulletin militaire de l'étranger (1871) ; etc. ${ }^{46}$ ). Enfin, on ne saurait oublier l'impact que joue le travail de constitution des plans de guerre dans cette même dynamique. Effectivement, c'est également à partir du XIX et du début du XX ${ }^{\mathrm{e}}$ siècle que les états-majors des grands Etats européens commencent à préparer systématiquement des plans de guerre pour l'éventualité des conflits avec leurs voisins (le plus populaire d'entre eux étant certainement le plan Schlieffen par lequel les forces armées allemandes initieront les opérations militaires de la Première Guerre mondiale) ${ }^{47}$. Ces plans, en grande partie élaborés sur un mode intertextuel bureaucratique (mais éga-

43. Latour B., Nous n'avons jamais été modernes, op.cit., p. 91.

44. Bucholz A., Moltke, Schlieffen, and Prussian War Planning, New York, Berg, 1991 ; Reardon C., Soldiers and Scholars - The U.S. Army and the Uses of Military History, 1865-1920, Lawrence, University Press of Kansas, 1990 ; Griffith P., Military Thought in the French Army 1815-1851, Manchester, Manchester University Press, 1989 ; Nenninger T. K., The Leavenworth Schools and the Old Army, Education, Professionalism, and the Officer Corps of the United States Army, 1881-1918, Westport et Londres, Greenwood, 1978 ; Bond B., The Victorian Army and the Staff College 1815-1914, Londres, Eyre Methuen, 1972 ; Luvaas J., The Education of an Army - British Military Thought 1815-1940, Chicago, University Press of Chicago, 1964.

45. Dodier N., L'expertise médicale - op.cit., p. 145.

46. Gat A., The Origins of Military Thought, op.cit., pp. 64-65. Voir également : Goltz C. von der, Rosbach et Iéna - Recherches sur l'état physique et intellectuel de l'armée prussienne pendant l'époque de transition du XVIII' au XIXe siècle, (trad. de l'allemand), Paris, Hinrichsen, 1890, p. 265 ; Dany J., "La littérature militaire d'aujourd'hui”, La Revue de Paris, avril 1912, pp. 611-624 ; Griffith P., Military Thought in the French Army, op.cit., p. 62. 
lement à l'aide de simulations opérationnelles et de nombreuses discussions), comportent entre autres des éléments relatifs à la logistique ainsi qu'aux moyens nécessaires pour mobiliser et déplacer les centaines de milliers de soldats alors enrôlés dans les armées allemande, autrichienne, états-unienne, française ou encore russe. Bien entendu, la constitution de ces plans militaires contribue très largement à la formation et au renforcement du régime d'expertise stratégique. On notera aussi que nombre des dynamiques bureaucratiques évoquées dans ce passage reposent sur l'effacement des traces de la constitution du même régime. Dans la réalisation des règlements, des manuels ou des plans, l'institution tend à faire disparaître la présence des individus (ainsi, nombre de textes sont anonymes) qui fondent le régime d'expertise pour leur substituer la personnalité d'une institution aux atours monolithiques et difficile à remettre en question sur un mode anthropologique ${ }^{48}$. Par ce biais aussi, on voit surgir une pensée stratégique de moins en moins « artisanale » mais toujours rattachée au cadre interprétatif classique.

Enfin, il faut noter que la majorité des traités militaires publiés à l'époque ne sont nullement secrets (à quelques exceptions près, telles que des textes du souverain Frédéric II qui seront néanmoins assez rapidement divulgués ${ }^{49}$ ). Il en découle qu'en dépit de la nationalisation des forces armées, surtout à partir du XIX siècle, l'expertise stratégique circule largement à travers l'Europe et en dehors de celle-ci. De longue date, les ouvrages militaires voyagent à travers l'Europe d'abord, dans le reste du monde ensuite. Les traductions de ces ouvrages sont par ailleurs de plus en plus nombreuses. Il est vrai que les officiers sont également habitués à voyager pour se former ou pour aller former des confrères. Ils font ainsi circuler le savoir stratégique. Les armées envoient par ailleurs ponctuellement des observateurs au sein de forces armées au combat. Ceux-ci sont parfois à l'origine de retours d'expérience publiés sous forme de livres, de rapports ou d'articles (ainsi, les officiers européens s'intéressent par exemple à la guerre civile américaine, même si les leçons qu'ils en tirent restent plutôt limitées à des questions très techniques) ${ }^{50}$. Les militaires peuvent aussi suivre indirectement des opérations par leurs lectures de la presse. Enfin, les officiers publient régulièrement des comptes rendus d'opérations étrangères dans les revues spécialisées. Le maillage intertextuel connaît donc aussi une diffusion de type transnational.

47. Pour plus de détails sur les plans de guerre, voir : Kennedy P. M., War Plans of the Great Powers, 1880-1914, Londres, Allen \& Unwin, 1979; Tunstall G. A., Planning for War against Russia and Serbia - Austro-Hungarian and German Military Strategies, 1871-1914, Columbia University Press, Boulder, 1993 ; Zuber T., Inventing the Schlieffen Plan German War Planning 19871-1914, Oxford, Oxford University Press, 2002. On notera cependant que l'existence d'un véritable plan Schlieffen est remise en question par certains historiens.

48. Boltanski L., De la critique - Précis de sociologie de l'émancipation, Paris, Gallimard, 2009.

49. Luvaas J. (dir. et trad. par), Frederick the Great on the Art of War, New York et Londres, The Free Press, 1966.

50. Luvaas J., The Military Legacy of the Civil War - The European Inheritance, Chicago, University of Chicago Press, 1959. 
Dans ses travaux sur la mémoire et l'histoire, Paul Ricœur évoquait l'existence d'une "mémoire-habitude », relativement spontanée, et d'une "mémoire obligée » qui repose sur le dressage des esprits ${ }^{51}$. A la lueur des éléments présentés jusqu’à présent, le régime d'expertise stratégique, qui repose sur la connexion des expériences par voie intertextuelle, correspond davantage à une mémoire d'obligation. Bien entendu, cet état de fait est en grande partie dû à l'enracinement institutionnel de ce savoir. Néanmoins, en dépit de l'existence de mécanismes complexes, et plus ou moins fortement institutionnalisés, de dressage et répétition, l'édifice stratégique, comme on va le voir, n'est pas à l'abri de la critique.

\section{Des épreuves de validité}

Effectivement, l'expertise stratégique est régulièrement soumise à des critiques de provenances diverses (et dont, encore une fois, seules les modalités intertextuelles seront prises en compte ici) qui sont autant d'épreuves auxquelles elle doit faire face. Ainsi, de la seconde moitié du XIXe siècle au XXe siècle, en particulier dans le contexte de la guerre de Sécession, des deux guerres des Boers, de la guerre russo-japonaise, des deux guerres mondiales ainsi que lors de la guerre froide, le régime d'expertise stratégique a affronté trois grandes catégories de critique auxquelles il a répondu selon des manières distinctes ${ }^{52}$.

Tout d'abord, le régime d'expertise a été confronté à des critiques qu'il s'est permis de traiter par le silence (ce qui constitue formellement, il est vrai, un rejet d'épreuve digne de ce nom). Cette attitude semble pouvoir s'expliquer de deux manières ${ }^{53}$. (a) Dans un premier cas, la critique, pour peu que son existence soit matériellement connue, ce qui est loin d'être toujours évident, est jugée non pertinente; elle ne mérite pas d'être prise en considération car sa voix est considérée comme trop discordante sur le fond et/ou trop incompatible sur la forme. Ce point est bien illustré par le rejet habituel des contestations se fondant, de manière assumée, sur l'expression de subjectivités, comme dans les romans (à cet égard, on peut songer à Le feu de Henri Barbusse ou $A$ l'Onest rien de nouveau de Erich Maria Remarque), le champ de la subjectivité s'avérant difficilement conciliable avec le biais techniciste du régime d'expertise. Il existe par ailleurs, on ne s'en étonnera guère, un rejet traditionnel, qui a rapport avec le fond de l'argumentation, des critiques radicales formulées par les pacifistes (on peut d'ailleurs étudier extensivement la pensée stratégique sans rencontrer le point de vue de ceux-ci). Ces rejets tendent à prouver que le régime d'expertise stratégique jouit d'un minimum d'autonomie. (b) Bien que cela soit difficile à prouver explicitement, on peut aussi suspecter que

51. Ricœur P., La mémoire, l'histoire, l'oubli, Paris, Seuil, 2000, p. 70 et p. 105 et suiv.

52. Boltanski L., De la critique - op.cit. ; Latour B., "Portrait de Gaston Lagaffe en philosophe technique", in Petites leçons de sociologie des sciences, Paris, La Découverte, 2006, pp. 15-24.

53. Dodier N., L'expertise médicale - op.cit., p. 228 et suiv. 
l'édifice stratégique refuse de prendre en considération certaines critiques non pas pour des raisons d'éloignement des points de vue, mais pour des motifs plus calculateurs ; prendre en considération une critique, c'est reconnaitre l'existence et une pertinence potentielle à cette critique. Bref, c'est se faire l'écho et donner du poids à cette critique. Quoi qu'il en soit, dans les deux cas évoqués, il y aurait donc refus de l'épreuve ouverte, ce que peut se permettre le régime d'expertise stratégique lorsqu'il est suffisamment confiant en lui. Notons cependant que, dans certaines situations, la critique peut être considérée comme très éloignée mais ne pas se heurter à ce fameux mur de silence. L'histoire de la pensée stratégique ne contient néanmoins pas pléthore d'exemples en la matière. Le cas le plus exemplaire reste probablement celui du banquier libéral et pacifiste polonais Jean de Bloch. Bloch avait fait publier en 1898 une somme posant la question de la possibilité de la guerre ${ }^{54}$. Il entrevoyait la difficulté de mener encore des guerres à une époque où les forces destructrices s'étaient très largement accrues. L'intérêt que la pensée stratégique a pu nourrir à son égard reposait certainement sur la capacité de cet analyste civil à interpréter des données militaires techniques. D'où l'utilité pour les militaires d'aborder le questionnement de Bloch et, ensuite, d'en relativiser la portée critique.

Dans un second temps, le régime d'expertise peut être soumis à des critiques internes, c'est-à-dire des critiques qui sont formulées dans le cadre reconnu par l'édifice. Ces critiques, qui se transforment régulièrement en controverses, ne peuvent simplement être passées sous silence au risque de voir naître au sein du régime d'irrémédiables incohérences qui pourraient, en dernière instance, remettre en question son existence même ${ }^{55}$. Ainsi, de la seconde moitié du XIXe siècle jusqu'à la veille de la Seconde Guerre mondiale, on a assisté à des controverses sur le degré d'initiative qu'il convenait de laisser aux commandants des grandes formations militaires en campagne (Bernhardi, Boguslawski, Freytag-Loringhoven, von de Goltz, etc.), sur l'emploi de dispositifs d'infanterie compacts ou dispersés dans un contexte d'augmentation de la puissance de feu (Ardant du Picq, Bonnal, Foch, etc.) ou encore sur l'emploi de blindés et de moyens aériens opérant de manière indépendante ou subordonnée aux autres armes (de Gaulle, Douhet, Fuller, Kingston-McCloughtry, Martel, Mitchell, Volckheim, etc.) ${ }^{56}$. Lorsque l'on suit attentivement ces controverses, dont le contenu précis est hors du propos de cette analyse, on remarque que l'on n'y croisera pas l'option de l'impossibilité de l'emploi de la force en temps de guerre. Ces controverses opèrent en effet comme un moyen de maintenir la critique dans les droits rails de l'orthodoxie stratégique. Plus encore, à défaut de faire naitre des remises en question

54. Echevarria A. J., After Clausewitz - German Military Thinkers Before the Great War, Lawrence, University Press of Kansas, 2000, p. 85 et suiv. ; Travers T., "Technology, Tactics, and Morale: Jean de Bloch, the Boer War, and British Military Theory", The Journal of Modern History, vol. 51, juin 1979, pp. 264-286.

55. Latour B., La science en action - op.cit., pp. 87-111. 
révolutionnaires de la pensée stratégique, ces controverses font apparaître l'existence de soupape de sécurité intellectuelle. Les acteurs des débats ne cessent en fait de répéter que la stratégie «c'est possible à condition que ... ». Et ce «à condition que ... » se traduit dans leurs propos par une réflexion sur des moyens d'ajustement technique. Le discours consiste alors à affirmer que : c'est possible à condition d'améliorer le moral des fantassins pour les inciter à monter à l'assaut de la tranchée ennemie (même si cette dernière est défendue par des mitrailleuses), c'est possible à condition d'employer les blindés de façon concentrée plutôt qu'en seul soutien de l'infanterie, c'est possible à condition d'attaquer par le flanc et non de front du dispositif général ennemi, c'est possible à condition de pilonner préalablement l'ennemi jusqu'à ce que son moral soit laminé, etc. En définitive, ces discussions tendent à faire du régime d'expertise un noyau dur, point de départ, accepté de tous lors des controverses, entouré de couches plus ou moins molles au sein desquelles se déroulent les joutes intellectuelles d'où surgissent divers ajustements. On notera par ailleurs qu'une part importante de ces ajustements, en particulier au XXe siècle, sont relatifs à l'intégration d'éléments matériels (armes automatiques, chars ou avions de combat, hélicoptères, etc.). Une fois encore, les controverses matérielles ne soulèvent pas la question de "savoir si » la stratégie est encore possible lorsque l'on dispose, par exemple, de mitrailleuses, mais bien de "savoir comment » faire de la stratégie lorsque de telles armes sont employées. En résumé, la question de l’impossibilité stratégique est soigneusement écartée dans les controverses militaires. Au final, ces controverses n'affaiblissent pas sérieusement le régime d'expertise stratégique. On peut plutôt penser qu'elles contribuent au renforcement de ce dernier grâce à la densification de la littérature stratégique se connectant à l'édifice intertextuel préexistant. En d'autres termes, les acteurs de la controverse, malgré leurs désaccords particuliers, ne cessent de réaffirmer leur croyance dans le régime d'expertise chaque fois qu'ils publient un document sur l'emploi des mitrailleuses, des chars, de l'aviation, des gaz de combat ou même, parfois, des armes nucléaires. Considéré sous cet angle, on peut aussi appréhender le régime d'expertise comme une sorte d'instance juridique suprême qui contraint les acteurs à penser d'une manière qui est tout sauf libre car ces derniers ne peu-

56. Voir à ce propos : Johnson D. E., Fast Tanks and Heavy Bombers - Innovation in the US Army, 1917-1945, Cornell, Cornell University Press, 2003 ; Habeck M., Storm of Steel-The Development of Armor Doctrine in Germany and the Soviet Union, 1919-1939, Ithaca et Londres, Cornell University Press, 2003 ; Frieser K-H., The Blitzkrieg Legend - The 1940 Campaign in the West, (trad. de l'allemand), Annapolis, Naval Institute Press, 2005 ; Echevarria A. J., After Clausewitz - op.cit. ; Winton H. R. et Mets D. R. (dir.), The Challenge of Change - Military Institutions and New Realities, 1918-1941, Lincoln et Londres, University of Nebraska Press, 2000 ; Gudmundsson B. I., Stormtroop Tactics - Innovation in the German Army, 1914-1918, Wesport, Praeger, 1995 ; Griffith P., Battle Tactics of the Western Front - The British Army's Art of Attack 1916-18, Londres et New Haven, Yale University Press, 1994 ; Corum J. S., The Roots of Blitzkrieg - Hans von Seeckt and German Military Reform, Lawrence, University Press of Kansas, 1992 ; Larson R. H., The British Army and the Theory of Armored Warfare 1918-1940, Newark, University of Delaware Press, 1984. 
vent jamais dire publiquement : «c'est impossible». Peu importent les chiffres des victimes, l'ampleur des destructions matérielles, les effets déstabilisants sur le plan social, économique ou psychologique (ou encore, plus simplement, l'opinion de l'homme d'infanterie pris individuellement et qui pense que c'est effectivement impossible) le consensus établi doit être maintenu.

Aux côtés des critiques provoquant des ajustements en interne, il existe également des critiques conduisant à d'impératifs recadrages quant à l'étendue du domaine d'expertise. Les critiques en question affirment que la guerre est en effet possible mais à condition d'étendre ou de réduire son domaine d'emprise. (a) Ainsi, dans le premier cas de figure, l'extension du domaine de validité de la guerre pourra mener à repenser le fonctionnement politique, social, économique, scientifique, moral, etc. des sociétés. Le discours stratégique engage donc bien plus que la seule communauté stratégique. Historiquement, on retrouve un exemple de ce phénomène dans la pensée stratégique allemande du début du XXe siècle avec des auteurs tels que Bernhardi, von der Goltz et, surtout, Ludendorff lorsqu'il s'exprime sur la guerre totale ${ }^{57}$. En dépit de la catastrophe humaine, sociale et politique qu'a constituée la Première Guerre mondiale, Ludendorff ne remet jamais en question l'option stratégique. En lieu et place, il ne cesse de fustiger le manque de volonté de tout ce qui n'est pas militaire. Le message est clair : la guerre est toujours possible à condition que l'ensemble de la société (le regard de Ludendorff porte en particulier sur le manque de volonté des civils) se plie à la logique stratégique. Autrement dit, chez Ludendorff et ses disciples, le maintien du régime stratégique est au prix du militarisme. Il implique donc une sérieuse remise en question de l'ensemble de la société. (b) Il est exact que le cas de figure inverse est également possible. Des auteurs, reconnus ou non comme membres de la communauté d'expertise, peuvent interpeller le régime en vue de proposer un recadrage réduit de son domaine de validité. Ainsi, suite à la Première Guerre mondiale, nombre de commentateurs se sont attachés à expliquer que l'emploi rationnel et instrumental de la force, «c'est possible, à condition que l'on revoie nos attentes à la baisse ». Plus concrètement, pour de nombreux stratégistes, la continuité du régime d'expertise impliquait d'envisager la guerre sous un jour plus défensif ou plus dissuasif 58. Pour certains de ces commentateurs, de manière caricaturale, on pouvait donc continuer à penser les opérations militaires, mais seulement derrière une ligne Maginot ou grâce à la présence d'une menaçante flotte aérienne. Une telle attitude est également perceptible lors de la guerre froide en ce qui concerne les armes nucléaires. L'apparition de ces dernières tend à réduire la validité de l’option stratégique, mais sans jamais en

57. Voir : Aron R., Penser la guerre, Clausewitz, op.cit.

58. Odom W. O., After the Trenches - The Transformation of U.S. Army Doctrine, 1918-1939, College Station, Texas A\&M University Press, 1999 ; Doise J., Vaïsse M., Politique étrangère de la France - Diplomatie et outil militaire 1871-1991, Paris, Imprimerie nationale, 1992 ; Higham R., The Military Intellectuals in Britain 1918-1939, New Brunswick, Rutgers University Press, 1966. 
contester les assomptions centrales 59. Les stratégistes nucléaires (Brodie, Halperin, Kaufmann, Kissinger, Osgood par exemple) ne remettent pas en question la validité technique du régime stratégique. Ce que certains d'entre eux mettent en évidence (en particulier parmi les stratégistes de l'OTAN), c'est le fait que celui-ci risque de mener à un échange nucléaire généralisé entre l'Est et l'Ouest qui rendrait non pertinent l'option stratégique classique. Pour ces mêmes stratégistes, l'option stratégique classique reste valable mais à certaines conditions, par exemple le fait de mener des guerres qu'ils nomment limitées. Pour terminer, notons également que l'on peut considérer les textes juridiques ou métajuridiques enjoignant le régime d'expertise stratégique à ne pas recommander le recours à tel ou tel armement (les gaz de combat, les armes bactériologiques, les mines anti personnelles, etc.) pour des raisons d'humanité sous le même angle ${ }^{60}$. Ces discours visent également à limiter la zone d'action du régime d'expertise sans en contester le cœur. Autrement dit, la réussite de l'épreuve critique peut donc impliquer, pour le régime d'expertise, une réduction de son domaine de validité.

En définitive, si l'on suit attentivement les évolutions historiques du régime d'expertise stratégique, on sera forcé d'admettre qu'il s'avère non seulement conservateur mais aussi très habile quant à sa capacité à faire face aux épreuves imposées par la critique. Ce régime est certes prêt à beaucoup débattre, mais la discussion ne remet guère en question ses affirmations de base (quel qu'en soit parfois le prix pour la société comme l'ont par exemple montré les deux guerres mondiales), relatives à l'usage technique de la force.

Cet article a cherché à succinctement mettre en évidence les principaux mécanismes fondateurs du régime d'expertise stratégique entre la fin du Moyen Age et la guerre froide. Ce régime trouve son origine dans un édifice intertextuel qui contribue à donner sa forme à et à définir la guerre. Plus encore, ce régime participe largement à la publicité de l'idée selon laquelle « la stratégie, ça fonctionne ». Ce faisant, il joue aujourd'hui encore un rôle essentiel dans les négociations sociales qui peuvent mener à la décision de partir en guerre. On dira donc qu'il constitue un point d'appui conventionnel nécessaire à l'élaboration d'un consensus sur le sujet et qu'il normalise ainsi la pratique guerrière. On notera aussi que la solidité du régime en question est ren-

59. Heuser B., NATO, Britain, France and the FRG: Nuclear Strategies and Forces in Europe 1949-2000, Londres, MacMillan, 1997 ; Steiner B. H., Bernard Brodie and the Foundations of American Nuclear Strategy, Lawrence, University Press of Kansas, Lawrence, 1991 ; Baylis J., Garnett J. (dir.), Makers of Nuclear Strategy, Londres, Pinter, 1991 ; Herken G., Counsels of War, New York, Oxford University Press, 1987 ; Kaplan F., The Wizards of Armageddon, New York, Touchstone, 1983 ; Freedman L., The Evolution of Nuclear Strategy, Londres, McMillan Press, 1981 ; Garthoff R. L., La doctrine militaire soviétique, (trad. de l'américain), Paris, Plon, 1952.

60. Best G., Humanity in Warfare, New York, Columbia University Press, 1980. 
forcée chaque jour par les décisions politiques, tacites ou explicites, de maintenir les forces armées et leurs budgets. Par ce biais, une caution est apportée à l'ensemble de l'édifice. Ajoutons encore que ce régime d'expertise est loin d'être monolithique. Il est traversé de nombreux conflits, que nous n'avons pas assez soulignés, entre auteurs et écoles de pensée. Néanmoins, comme cela a été suggéré, ces oppositions ne remettent jamais complètement en cause la possibilité technique que constitue l'option stratégique. De ce point de vue, l'édifice fait preuve de capacités réflexives très limitées.

Enfin, il faut malgré tout se méfier de l'apport concret de ce régime d'expertise par rapport aux pratiques guerrières les plus précises. La finesse de la pensée stratégique écrite dans le confort des bureaux n'est pas toujours, loin s'en faut, en adéquation avec le vécu du terrain. Donc, il conviendra aussi de s'interroger sur la fidélité des porte-parole que sont les commentateurs stratégiques sur ce que vivent les militaires. Les catégories opérationnelles si nettes sur le papier peuvent ne pas résister à l'épreuve du champ de bataille. D'autre part, il faudra aussi se demander quel est le niveau de pertinence accordé par les acteurs de terrain à l'immense édifice intertextuel et ce que ces derniers en font dans leur quotidien guerrier (acceptation « en bloc », rejet partiel, adaptation plus ou moins importante). Il existe là matière à investigations à mener sur un mode plus anthropologique (et oral) en vue de comprendre ce qu'il en est des méthodes d'appropriation locale de l'édifice mis en évidence ${ }^{61}$.

61. Pour une réflexion théorique sur ce sujet, voir : Corcuff P., "Eléments d'épistémologie ordinaire du syndicalisme", Revue française de science politique, vol. 41, nº, août 1991, pp. 190209. Pour une application de la «méthode anthropologique » (au sens large) dans l'univers guerrier, voir par exemple : Thiéblemont A., Expériences opérationnelles dans l'armée de terre: unités de combat en Bosnie (1992-1995), 3 tomes, Les documents du C2SD n 42 , novembre 2001 ; Mauss-Copeaux C., Appelés en Algérie - La parole confisquée, Paris, Hachette, 1998 ; Doubler M., Closing with Enemy - How GIs Fought the War in Europe, 1944-1945, Lawrence, University Press of Kansas, 1994. 